\title{
Noise-Immune Conjugate Large-Area Atom Interferometers
}

\author{
Sven Herrmann, ${ }^{1}$ Sheng-wey Chiow, ${ }^{1}$ Steven Chu, ${ }^{1,2,3}$ and Holger Müller ${ }^{1,2,3, *}$ \\ ${ }^{1}$ Physics Department, Stanford University, 382 Via Pueblo Mall, Stanford, California 94305, USA \\ ${ }^{2}$ Department of Physics, 366 Le Conte Hall, University of California, Berkeley, CA 94720-7300 \\ ${ }^{3}$ Lawrence Berkeley National Laboratory, One Cyclotron Road, Berkeley, CA 94720.
}

(Dated: October 27, 2018)

\begin{abstract}
We present a pair of simultaneous conjugate Ramsey-Bordé atom interferometers (SCI) using large $(20 \hbar k)$-momentum transfer (LMT) beam splitters, where $\hbar k$ is the photon momentum. Simultaneous operation allows for common-mode rejection of vibrational noise. This allows us to surpass the enclosed space-time area of previous interferometers with a splitting of $20 \hbar k$ by a factor of 2,500 . Among applications, we demonstrate a $3.4 \mathrm{ppb}$ resolution in the fine structure constant and discuss tests of fundamental laws of physics.
\end{abstract}

Light-pulse atom interferometers can convert a small signal into a relatively large phase shift of the interference fringes. For example, in Ref. [1], a 3 parts per billion (ppb) modulation in local gravity leads to a $1 \%$ shift of the interference fringe. They thus make excellent microscopes for small signals that have been applied in many cutting-edge precision measurements [110]. Large-momentum transfer (LMT) beam splitters, which have become practical recently [11], promise to increase the sensitivity further, by factors of $10 \mathrm{~s}$ to $100 \mathrm{~s}$, by increasing the space-time area enclosed between the interferometer arms. But just as vibrations blur microscopic images, they blur the interference fringes in interferometers. This becomes more pronounced as the sensitivity is increased until eventually interferences can no longer be discerned. This has so far limited the use of LMT beam splitters to very short pulse separation times $T$ (Fig. 1) of $1 \mathrm{~ms}$, thwarting the potential gain in sensitivity.

Cancellation of vibrations has been demonstrated by using the same laser light to simultaneously address two similar interferometers at separate locations [12-14]. This method, however, is restricted to situations where the differential signal is small (in this case, the gravity gradient), so that the interferometers are similar enough to be addressable by the same laser.

In this work, we explore the full potential of LMT by cancelling vibrations between dissimilar interferometers, the conjugate Ramsey-Bordé interferometers shown in Fig. 1. The idea is to use laser pulses that contain a pair of frequencies whose phase noise is extremely well correlated. We use this to demonstrate a 2,500-fold increase in the enclosed space-time area of interferometers with 20 -photon momentum transfer, without a reduction in contrast. This paves the path towards strongly enhanced sensitivity in measurements of fundamental constants $[8,9,15]$, tests of general relativity [1] or the equivalence principle [10], and detection of gravitational waves [16].

Atom interferometers basically consist of a source of atoms and beam splitters for the matter waves. The atom source for our interferometers is a fountain of cesium

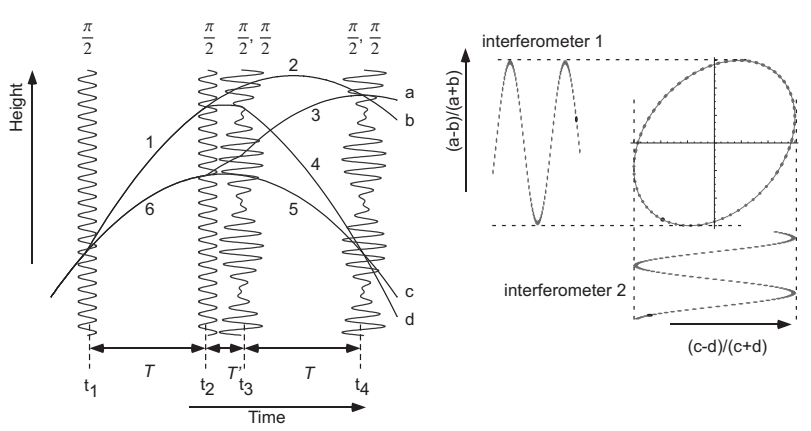

FIG. 1: Correlating the fringes of two interferometers creates an ellipse whose eccentricity allows to determine the relative phase.

atoms with a moving optical molasses launch and Raman sideband cooling in an optical lattice, as described in [17]. As beam splitters, we use multiphoton Bragg diffraction of matter waves at an optical lattice [11, 18-20]. The optical lattice is formed by two counterpropagating laser beams that we may call the top and bottom beam (Fig. 2). Bragg diffraction can be described in the initial rest frame of the atom. For example, the atom may absorb $n$ photons at $\omega_{1}$ from the bottom beam and be stimulated to emit $n$ at $\omega_{2}$ into the top beam. The atom emerges at the same internal quantum state with a momentum of $2 n \hbar k$, where $k$ is the wavenumber, and a kinetic energy of $(n \hbar k)^{2} /(2 M)$, where $M$ is the mass of the atom. This energy has to match the energy $n \hbar\left(\omega_{1}-\omega_{2}\right)$ lost by the laser field, which allows us to choose the Bragg diffraction order $n$ by the difference frequency $\omega_{1}-\omega_{2}$.

Fig. 1 (left) shows a space-time diagram of our Ramsey-Bordé interferometers. Let us specialize to the lower one, whose outputs are labelled $\mathrm{c}$ and $\mathrm{d}$. An atom enters on its way upwards. At a time $t_{1}$, a " $\pi / 2$ " laser pulse transfers a momentum of $2 n \hbar k$ with a probability of $50 \%$. Depending on whether momentum was transferred or not, the atom follows trajectory 1 or 6 . At $t_{1}+T$, a second $\pi / 2$ pulse stops the relative motion of them. After two more pulses, the paths are recombined into the outputs c and d where they interfere. A second, 
upper, interferometer is formed by recombining the other outputs of the beam splitter at $t_{2}$.

The probability that the atom arrives at output c, for example, is given by $\cos ^{2} \phi$, where $\phi=\phi_{F}+\phi_{I}$ is the phase difference of the interferometer arms when they interfere. This contains a contribution $\phi_{F}$ of the atom's free evolution between the beam splitters and one of the interaction with the light $\phi_{I}$. The free evolution phase $\phi_{F}=S_{\mathrm{Cl}} / \hbar$ is given by the classical action $S_{\mathrm{Cl}}=\int\left(E_{\mathrm{kin}}-E_{\mathrm{pot}}\right) d t$, where $E_{\text {kin }}$ and $E_{\mathrm{pot}}$ are the kinetic and potential energy. The interaction phase $\phi_{I}$ is because whenever a photon is absorbed, its phase is added to the matter wave phase and subtracted for emission of a photon [21]. This phase is different for the two paths because of the respective spatial separation of the interactions at $t_{2}$ and $t_{3}$. Summing up, $[8,11]$

$$
\phi^{ \pm}= \pm 8 n^{2} \omega_{r} T+2 n k g\left(T+T^{\prime}\right) T+n \phi_{L}^{ \pm},
$$

where $\omega_{r}=\hbar k^{2} /(2 M)$ is the recoil frequency and $g$ the local gravitational acceleration. The plus and minus signs are for the upper and lower interferometer, respectively, and $\phi_{L}^{ \pm}=\phi_{2}-\phi_{1}-\phi_{4}^{ \pm}+\phi_{3}^{ \pm}$is given by the phases $\phi_{1-4}$ of the laser pulses at $t_{1-4}$. This equation shows that LMT beam splitters can increase the sensitivity of the phase towards gravity by a factor of $n$ and the one towards the recoil by $n^{2}$.

Because of the motion of the atoms, which gives rise to a Doppler frequency shift, addressing the upper and lower interferometer requires two separate laser frequencies $\omega_{2}, \omega_{3}$ in the top beam. The phases of these respective frequencies are denoted $\phi_{3}^{ \pm}$and $\phi_{4}^{ \pm}$.

The influence of vibrations is because the atoms define a freely falling inertial frame. Any vibrations of the laboratory translate into phase shifts of the laser beams in this frame, and if the distribution of $\phi_{L}$ has a width that is comparable to $\pi / n$, they render the interferences invisible. With non-LMT beam splitters, vibrations can be suppressed to acceptable levels by state of the art vibration isolation [22]. This becomes difficult, however, with $n \gg 1$ LMT beam splitters.

The idea underlying the cancellation of vibrations is to run the upper and lower interferometers simultaneously ("simultaneous conjugate interferometers," SCIs) and to use

$$
\Phi \equiv \phi^{+}-\phi^{-}=16 n^{2} \omega_{r} T+n \phi_{L}
$$

where

$$
\phi_{L}=\left(\phi_{3}^{+}-\phi_{3}^{-}\right)+\left(\phi_{4}^{-}-\phi_{4}^{+}\right)
$$

depends only upon the difference between laser phases at the last two beam splitters. Thus, the requirement of absolute phase stability in one individual interferometer has been reduced to one of relative stability between two: if $\delta \phi_{L}=0$, the overall phase $\Phi=$ const., independent of vibrations. Then, their fringes as plotted in Fig.

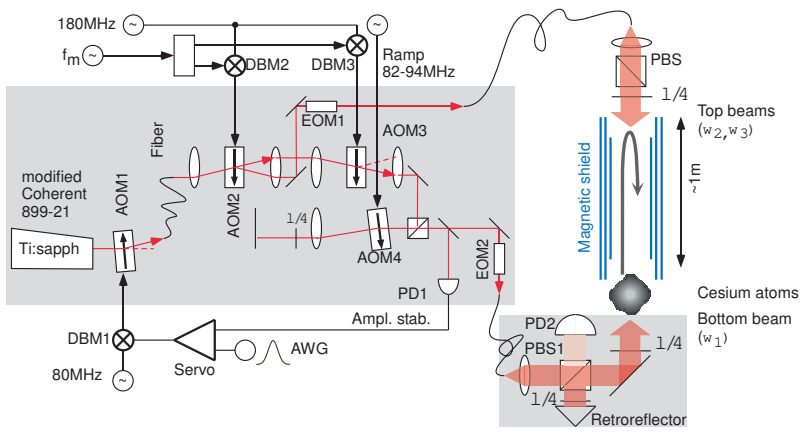

FIG. 2: Setup.

1 form an ellipse. The common phase moves the data points around the ellipse, but the differential phase can be extracted by ellipse-specific fitting. One way that has been realized, without LMT [12-14], is to use the same radiation to address both interferometers, which trivially leads to $\delta \phi_{L}=0$. However, the use of this method is restricted to measurements of very small differential signals, so that the interferometers are similar; whereas our SCIs are dissimilar and can be sensitive to the relatively large $16 n^{2} \omega_{r} T$ differential signal.

One essential requirement for the experimental setup (Fig. 2) is to satisfy $\delta \phi_{L}=0$ as well as technically possible. Moreover, our laser system is optimized for driving LMT beam splitters based on high-order Bragg diffraction $[11,20]$, which requires laser pulses having smooth envelope functions with an optimized duration and high power.

The laser light originates from a $6 \mathrm{~W}$ injection-locked titanium:sapphire laser at $852 \mathrm{~nm}$ wavelength [11, 23]. Its frequency is referenced to the $F=3 \rightarrow F^{\prime}=2 \mathrm{D} 2$ line of a modulation transfer spectroscopy in a Cs vapor cell (not shown); an offset of up to $\pm 20 \mathrm{GHz}$ can be set by means of an offset lock. For intensity control and forming the Gaussian envelope functions of the beam splitting-pulses, we use an acousto-optical modulator AOM1 within a feedback loop, see Fig. 2.

As a result of the different velocities, the resonance conditions for the third and fourth beam splitter are shifted by $16 n \omega_{r}$ between the two interferometers. To satisfy both, AOM2 is driven by two rf signals of equal amplitude at frequencies of $180 \mathrm{MHz} \pm f_{m}$. It thus generates two optical frequencies in its deflected output that differ by $2 f_{m}$. They follow the same optical path; thus, length fluctuations such as caused by vibrations, air currents, etc., are common-mode and do not degrade the phase noise in the difference frequency. We have previously shown that a phase variance of $\sigma^{2} \approx(160 \mu \mathrm{rad})^{2}$ is possible [24]. The power in each component is set to $1 / 8$ of the power at the input of AOM2, which maximizes the Rabi frequency of driving the atoms.

To generate the counterpropagating beam, we use the undeflected power from AOM2. Use of this radiation, 
which would otherwise be lost, allows us to make good use of our available laser power. This, however, varies between $1 / 2$ and 1 of AOM2's input power at the beat frequency $2 f_{m}$ of the two rf signals. AOM3 is used to take out this undesired modulation. It is driven by a "conjugate" rf signal, which is strong when the rf drive of AOM2 is weak and vice versa. The amplitude modulation is thus suppressed - residual sidebands are below 0.0018 (or $-27 \mathrm{~dB}$ ) of the carrier power.

Due to the free fall of the atoms, the resonance condition in the laboratory frame changes at a rate of about $23 \mathrm{MHz} / \mathrm{s}$. We account for this by ramping the frequency of the bottom beam using the double-passed AOM4.

The beams are brought to the experiment via singlemode, polarization-maintaining fibers and collimated to an $1 / e^{2}$ intensity radius of about $3 \mathrm{~mm}$ by a commercial fiber port (Thorlabs, Inc., Newton, NJ) or $12.5 \mathrm{~mm}$ by a triplet lens, a combination of an aplanatic meniscus (CVI Melles Griot 01 LAM 225/076) and an achromatic doublet (Thorlabs AC508-200B). The polarizations are made circular to $\left(\sigma^{+}-\sigma^{+}\right)$by zeroth-order quarter wave retardation plates. The bottom beam can have a maximum power of $1.15 \mathrm{~W}$ at the fiber output; the top beam a peak power of $1.6 \mathrm{~W}$, i.e., $0.4 \mathrm{~W}$ per frequency. Alternatively, we overlap both beams at a polarizing beam splitter and send them through the same fiber with orthogonal polarizations. The upper fiber collimation optics is then replaced by a hollow corner-cube retroreflector and a quarter wave plate. This method simplifies beam alignment. Also, it was found important to shield the beams from air currents to prevent a loss of contrast.

The first interferometer pulse is typically applied at $t_{1}=70 \mathrm{~ms}$ after launch. For $20 \hbar k$ beam splitters, we use a detuning of about $3-4 \mathrm{GHz}$ and peak intensities of $0.4 \mathrm{~W} / \mathrm{cm}^{2}$ in the bottom beam and $0.13 \mathrm{~W} / \mathrm{cm}^{2}$ per frequency in the top beam with a waist of $12.5 \mathrm{~mm}$; with thin $\left(w_{0}=3 \mathrm{~mm}\right)$ beams a detuning of $16 \mathrm{GHz}$ is used. After elapse of the full interferometer sequence, the atoms in the four interferometer outputs a-d (Fig. 2, left) are separately detected by their fluorescence $f_{a-d}$ as they pass a photomultiplier tube in free fall. To take out fluctuations in the atom number, we define the normalized fluorescence $F_{u}=\left(f_{a}-f_{b}\right) /\left(f_{a}+f_{b}\right)$ of the upper interferometer and $F_{l}$ in analogy for the lower interferometer.

Fig. 3 shows examples for ellipses measured by our SCIs at a short pulse separation time of $T=1 \mathrm{~ms}$. A contrast of around $25-31 \%$ is achieved at momentum transfers between $(8-20) \hbar k$ (the theoretical optimum is $50 \%$, because each detected interferometer output overlaps spatially with population lost in the third beam splitter which does not interfere). It is evident that the strong dependence of the contrast upon the momentum transfer, that was observed in previous LMT interferometers [11], is absent. This is a first benefit of SCIs.

The dependence of the contrast on the pulse separation time $T$ is shown for $10 \hbar k$ and $20 \hbar k$ interferometers
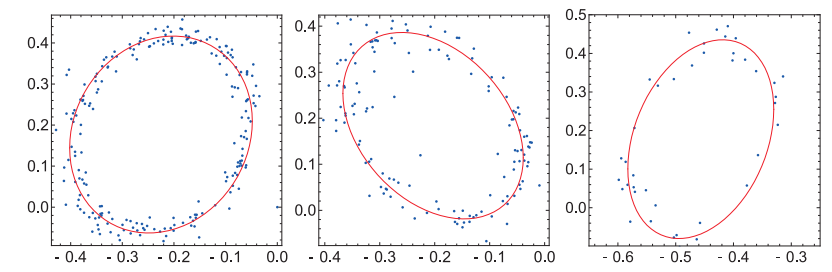

FIG. 3: Left: $12 \hbar k, 1 \mathrm{~ms}, 25 \%$ contrast. Middle: $14 \hbar k, 1 \mathrm{~ms}$, $25 \%$ contrast; Right: $20 \hbar k, 1 \mathrm{~ms}, 27 \%$.
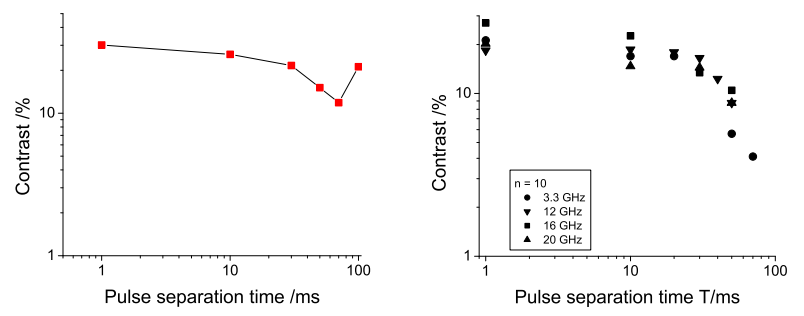

FIG. 4: Left: Contrast versus pulse separation time for a $10 \hbar k$ interferometer. The improvement at $T=100 \mathrm{~ms}$ was reached by improved optics and shielding from air currents (see Fig. 5 for the ellipse underlying this datum). Right: same for a $20 \hbar k$ interferometer with different detunings.

in Fig. 4. A certain decrease for long $T$ is expected because then a fraction of the atoms leave the area of the laser beams due to their thermal velocity. Nevertheless, a contrast of $21 \%$ can be obtained for $T=100 \mathrm{~ms}$ and $10 \hbar k$. For a $20 \hbar k$ device, contrast is $10 \%$ at $T=50 \mathrm{~ms}$. In previous work without SCIs, $8 \%$ contrast at $20 \hbar k$ was only possible at $T \leq 1 \mathrm{~ms}$ [11]. Thus, SCIs allow us to improve the pulse separation time to $50 \mathrm{~ms}$ from $1 \mathrm{~ms}$, without a reduction in contrast. This corresponds to a 2,500-fold increase in the enclosed space-time area.

Our dissimilar interferometers exhibit a large differential signal (the $16 n^{2} \omega_{r} T$ term due to the photon recoil), allowing for high-resolution measurements. To demonstrate this, Fig. 5 shows 2 data sets, each containing 1,300 data points taken with a $10 \hbar k$ interferometer pair having a pulse separation time of $T=100 \mathrm{~ms}$. To analyze the data, we use a Bayesian estimation [13], which shows a better immunity from systematic errors than simpler methods [25]. At a given signal to noise ratio, the phase $\Phi$ can be best determined if it is near $\pm \pi / 2$, when the ellipse is close to a circle. Therefore, an offset of $+\pi / 2$ and $-\pi / 2$, respectively, was used for the two measurements shown, and the laser frequency offset $f_{m}$ that yields $\Phi=0$ can be determined from the average of the phase estimates. From a total of 12,000 such points, we obtain a resolution of $6.8 \mathrm{ppb}$ within $7 \mathrm{~h}$ of measurement. Via Eq. 2, $\omega_{r}$ and thus $\hbar / M$ can be determined; correspondingly, our SCIs are sensitive to the fine structure constant $\alpha$ via $\alpha=\left[\left(2 R_{\infty} / c\right)\left(M / m_{e}\right)(h / M)\right]^{1 / 2}$, where $R_{\infty}$ is the Rydberg constant and $m_{e}$ the electron mass, to 


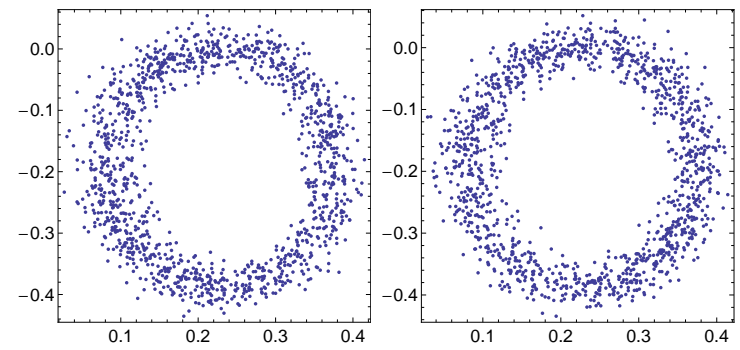

FIG. 5: Each graph shows 1300 data points $(n=5, T=$ $100 \mathrm{~ms}, C=20 \%$ ) taken at $\Phi=+\pi / 2$ and $-\pi / 2$, respectively.

a resolution of $3.4 \mathrm{ppb}$. Without SCIs and LMT, achieving a similar resolution would take several weeks' worth of data [8]. It is similar to interferometers using Bloch oscillation for $\sim 2000 \hbar k$ common-mode momentum transfer, where $88 \mathrm{~h}$ of data $(4 \times 221$ fringes that take 6 min each) yield 3 ppb resolution and $4.6 \mathrm{ppb}$ absolute precision [9]. This is because of (i) the $n^{2}$ scaling of the sensitivity with momentum transfer in our method, and (ii) cancellation of vibrational noise between SCIs. Further reduction of this statistical uncertainty and an analysis of systematic errors are beyond the scope of this Letter.

We remark that cancellation of vibrations is equally important in Mach-Zehnder interferometers (MZIs) with LMT. The adaption of our methods to this case is straightforward, as MZIs are slightly simpler, requiring three light pulses instead of four and featuring a $100 \%$ theoretical contrast. Many MZI applications gain sensitivity proportional to the enclosed area, which means that our work allows for a 2,500 fold improvement there. The vibration cancellation between LMT interferometers demonstrated here is also a crucial technology for the detection of gravitational waves [16].

The dissimilarity of the interferometers in this case could be external fields affecting the interferometer geometry, different atomic species, or even different laser wavenumbers $k_{1}$ and $k_{2}$. In the latter case, the phase relationship between these lasers could be established by a frequency comb. Cancellation of vibrations then requires that $k_{1} n_{1}$ and $k_{2} n_{2}$, where $n_{1,2}$ are the Bragg diffraction orders, satisfy a simple rational relationship. A Lissajous figure will then be generated which reduces to an ellipse for $k_{1} n_{1}=k_{2} n_{2}$. Bayesian estimation can be used to extract the phase. The possibility of correlating signals from different atoms are interesting for tests of the equivalence principle [10], and may allow new paths to cancel systematic effects in searches for an electron electric dipole moment [26], tests of charge neutrality [27], and other experiments.

In this work, we have presented common-mode rejection between dissimilar atom interferometers addressed by different overlapped laser frequencies. Compared to previous work [11], we demonstrate a 2,500-fold increase in the enclosed space-time area of atom interferometers using $20 \hbar k$ momentum transfer, without a reduction in interference contrast. By removing the most important limitation on the space-time area, and hence sensitivity, of such large momentum transfer interferometers, this work opens the door towards many exciting experiments.

S.H. and H.M. thank the Alexander von Humboldt foundation. This material is based upon work supported by the National Science Foundation under Grant No. 0400866 and by the Air Force Office of Scientific Research under Award Number FA9550-04-1-0040.

* Electronic address: hm@berkeley.edu;http://physics. berkeley.edu/research/mueller/

[1] H. Müller et al., Phys. Rev. Lett. 100, 031101 (2008).

[2] A. Peters, K.Y. Chung, and S. Chu, Nature (London) 400, 849 (1999); Metrologia 38, 25 (2001).

[3] T.L. Gustavson, A. Landragin, and M.A. Kasevich, Class. Quantum Gravity 17, 2385 (2000).

[4] D. S. Durfee, Y. K. Shaham, and M. A. Kasevich Phys. Rev. Lett. 97, 240801 (2006).

[5] B. Canuel et al., Phys. Rev. Lett. 97, 010402 (2006).

[6] J.B. Fixler et al, Science 315, 74 (2007).

[7] G. Lamporesi, et al.,Phys. Rev. Lett. 100, 050801 (2008).

[8] A. Wicht et al., Physica Scripta T102, 82 (2002).

[9] P. Cladé et al., Phys. Rev. Lett. 96, 033001 (2006); Phys. Rev. A 74, 052109 (2006); M. Cadoret et al., Phys. Rev. Lett. 101, 230801 (2008).

[10] S. Dimopoulos et al., Phys. Rev. Lett. 98, 111102 (2007); arXiv:0802.4098 (2008).

[11] H. Müller et al., Phys. Rev. Lett. 100, 180405 (2008).

[12] M.J. Snadden et al., Phys. Rev. Lett. 81, 971 (1998).

[13] J. K. Stockton, X. Wu, and M. A. Kasevich, Phys. Rev. A 76, 033613 (2007).

[14] A. Bertoldi et al., Eur. Phys. J. D 40, 271 (1996).

[15] H. Müller et al., Appl. Phys. B 84, 633 (2006).

[16] S. Dimopoulos, P. W. Graham, J. M. Hogan, M. A. Kasevich, and S. Rajendran, Phys. Rev. D 78, 122002 (2008); arXiv:0712.1250.

[17] P. Treutlein, K.Y. Chung, and S. Chu, Phys. Rev. A 63, 051401(R)

[18] D.M. Giltner, R.W. McGowan, and S.A. Lee, Phys. Rev. Lett. 75, 2638 (1995); Phys. Rev. A 52, 3966 (1995).

[19] A. Miffre et al., Eur. Phys. J. D 33, 99 (2005).

[20] H. Müller, S.-w. Chiow, and S. Chu, Phys. Rev. A 77, 023609 (2008).

[21] D. S. Weiss, B. C. Young and S. Chu, Appl. Phys. B 59, 217 (1994).

[22] J.M. Hensley, A. Peters, S. Chu, Rev. Sci. Instrum. 70, 2735 (1999).

[23] S.-w. Chiow, S. Herrmann, H. Müller, and S. Chu, to be published.

[24] H. Müller et al., Opt. Lett. 31, 202 (2006).

[25] G. T. Foster, J. B. Fixler, J. M. McGuirk, and M. A. Kasevich, Opt. Lett. 27, 951 (2002).

[26] C. Chin, V. Leiber, V. Vuletic, A. J. Kerman, and S. Chu, Phys. Rev. A 63, 033401 (2001).

[27] C. Lämmerzahl, A. Macias, and H. Müller, Phys. Rev. A 75, 052104 (2007); A. Arvanitaki et al, Phys. Rev. Lett. 100, 120407 (2008). 